\title{
Optimization for the cathodic protection system based on numerical simulation
}

\author{
Weiguo Zeng ${ }^{1}$, Dongxiao $\mathrm{Xu}^{2}$, Hao Wang ${ }^{2}$, Yong Yang ${ }^{1 *}$ and Hongmei Wang ${ }^{2}$ \\ ${ }^{1}$ China Special Equipment Inspection and Research Institute, Beijing, 100000,China \\ ${ }^{2}$ The First Gas Production Plant of Changqing Oilfield Branch, Xi'an, Shanxi, 71000,China
}

\begin{abstract}
Cathodic protection was one of important method for the corrosion prevention. With the expand of pipeline at Jingbian gas field, the cathodic protection system had been not qualified for the corrosion prevention of pipeline at Jingbian gas field. The results of survey for pipeline in 2018 years suggested that there were less-protection or over-protection for the corrosion prevention of pipeline. In this study, it was simulated by the COMSOL software to clear the regulation of current for pipeline in different cathodic protection area, and learn the overall situation of cathodic protection station in Jingbian gas field. Finally, optimizing the cathodic protection system for improving the effect by cathodic protection and ensuring the safety production.
\end{abstract}

\section{Introduction}

Cathodic protection was a technology to protect the metallic pipeline from corrosion by adding a negative potential, basing on the following theory:

$$
\mathrm{M}-\mathrm{ne}^{-} \rightleftharpoons \mathrm{M}^{\mathrm{n}+}, E_{\mathrm{h}}
$$

Where, the $E_{\mathrm{h}}$ was a negative potential. When the adding negative potential was lower than the negative potential of $E_{\mathrm{h}}$, the metallic pipeline was protected through an inhibition of corrosion. However, the protective effect would reduce when the adding negative potential was higher than $E_{\mathrm{h}}$.

As a change of energy structure, the requirement for petroleum and natural gas were increased further. This change would expand the scale of pipeline and enhance the corrosion prevention of pipeline. Specially, with the expand of pipeline at Jingbian gas field, the cathodic protection system had been not qualified for the corrosion prevention of pipeline at Jingbian gas field. And the most common problem was that the adding negative potential strayed away from the normal range because of the expansion of pipeline. Then, there was a phenomenon of less-protection or over-protection for the corrosion prevention of pipeline. Therefore, it was necessary to optimize the cathodic protection system for keeping the effective protection along with the expand of pipeline.

Recently, the numerical simulation had been used for the study of potential distribution of cathodic protection system due to the fast development of electrochemistry and computer simulation technology. The numerical simulation could precisely forecast the effect of cathodic protection, and solve the problems of less-protection or over-protection. Among the various computer simulation software, the COMSOL was most famous software platform, which could be used for modeling and simulating the physical field based on the advanced digit methodologies. What's more, the COMSOL software contained more than 30 modules, including the corrosion module, which could expand the function of modeling for the most physical field.

Herein, this study employed the COMSOL software, and divided the geometric model into irregular net through the finite element method. Furthermore, simulating the surficial potential distribution of model following the assumption of the reaction and boundary conditions, to learn the overall situation of cathodic protection station in Jingbian gas field. Finally, optimizing the cathodic protection system for improving the protection effect by cathodic protection and ensuring the safety production.

\section{Modeling}

The model contained two parts of physical model and electrochemical model. For the physical model, it was established according to the practical parameters of the length of pipeline, thickness of wall and anticorrosive coating, distance of anode, the buried depth and location of pipeline. While the electrochemical model with well-known equations was built based on the physical geometric model, combining the pipeline and electrochemical reaction of anode.

*Corresponding author's e-mail: yystanb $@ 163 . c o m$ 


\subsection{Physical geometric model}

This physical geometric model was consisted by three cathodic protection stations, locating at the Sanjing-South 32 in Jinbian gas field. The detail parameters were described as following:

Zhong 21 station: Size of pipeline was $219 \mathrm{~mm} \times 7 \mathrm{~mm}$, depth both for pipeline and assistant anode was $2 \mathrm{~m}$, distance between pipeline and assistant anode was $100 \mathrm{~m}$, resistivity of soils and expoxy-anticorrosive coating without defect were $188.4 \Omega \cdot \mathrm{m}$ and $4 \times 10^{5} \Omega \cdot \mathrm{m}^{2}$.

\subsection{Electrochemical model}

The steady state model of secondary current distribution was established by the Electrochemistry module in COMSOL software platform. Specially, the oxidation of water reacted on the surface of assistant anode, while the reduction of oxygen reacted on the surface of pipeline. Additionally, the limited current density on the surface of pipeline was taken into consideration due to the limitation of oxygen content in the soils. Therefore, the electrode kinetics could be expressed as following:

$$
\begin{aligned}
& \mathrm{i}_{\exp r}=-i_{0} \times 10^{\eta / A_{c}} \\
& \mathrm{i}_{l o c}=\frac{i_{\exp r}}{1+\left|\frac{i_{\exp r}}{i_{\text {lim }}}\right|}
\end{aligned}
$$

Where, $i_{0}$ was the exchange current density, $\mathrm{A} / \mathrm{m}^{2} ; \eta$ was the overpotential, $\mathrm{V} ; A_{\mathrm{c}}$ was the Tafel slope of cathode; $i_{\text {lim }}$ was the limited current density, $\mathrm{A} / \mathrm{m}^{2} ; i_{\text {expr }}$ was the current density without consideration of oxygen content, $\mathrm{A} / \mathrm{m}^{2} ; \quad i_{\text {loc }}$ was the current density with consideration of oxygen content, $\mathrm{A} / \mathrm{m}^{2}$.

It should be noted that the initial parameters for the establishing of secondary current distribution model were described as following:

$i_{0}=1.518 \times 10^{-5} \mathrm{~A} / \mathrm{m}^{2}$

$E_{\text {eq }}=-0.659 \mathrm{~V}$

$i_{\lim }=0.01 \mathrm{~A} / \mathrm{m}^{2}$

$I_{\mathrm{a}}=1.87 \mathrm{~A}$

\section{Comparison between numerical simulation and measurement}

To analyze the accuracy and reliability of COMSOL simulation, comparing the results of COMSOL simulation and the real potential distribution curves measured by the CIPS. Therefore, it was necessary to correct the model. In this study, we established the relationship between the degree of influence and the corrective coefficient of $K$, which was obtained by the modification for the 123 pipelines with a consideration of influencing factor and real states. And the $K$ was expressed as following:

$$
\begin{aligned}
& K=\alpha^{m} \\
& m=m_{1}+m_{2}+m_{3}+m_{4} \\
& V_{\text {corr }}=K\left(V_{\text {theo }}-\mathrm{e}\right)+\mathrm{e}
\end{aligned}
$$

Where, $K$ was the corrective coefficient, $\alpha$ was a correction factor of $0.921, m$ was the value of degree of influence, $e$ was the natural potential, $V_{\text {corr }}$ was corrected protection potential, and $V_{\text {theo }}$ was the theoretical protection potential.

The Figure 1 and Figure 2 were the protection potential result from the COMSOL simulation based on a corrected model, and the real protection potential result from CIPS measurement for the Zhong 24 to Zhong 8 pipelines, respectively. It could be noted that the pipeline was in a state of less-protection both for results of simulation and measurement. And the tendencies of protection potential for simulation and measurement were the same with a minor error of $10 \sim 20 \mathrm{mV}$.

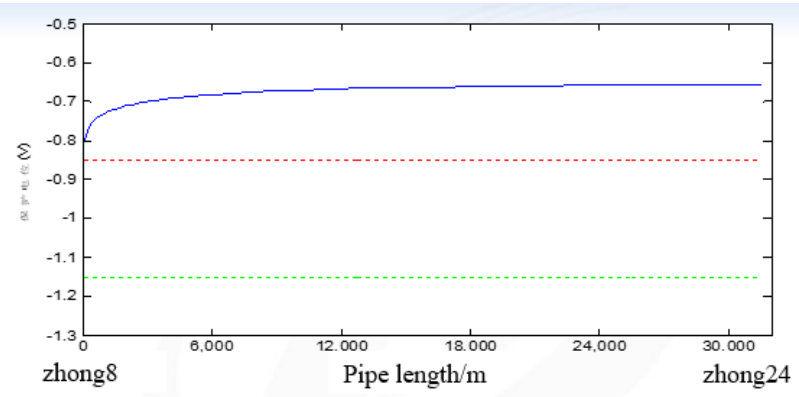

Figure 1. Less-protection by the protection potential result from COMSOL simulation

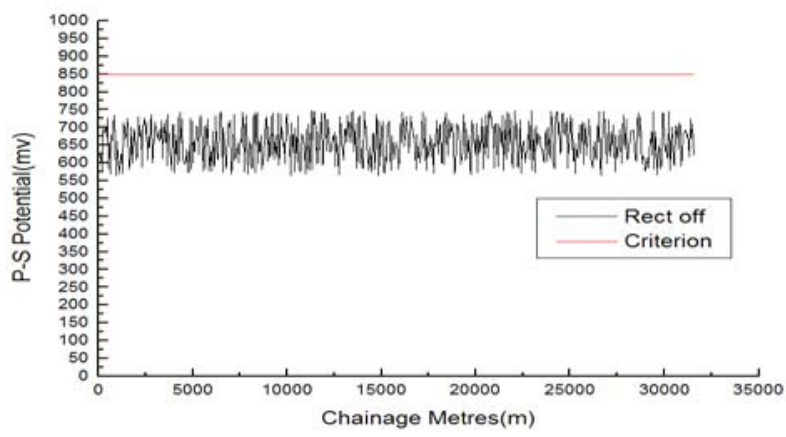

Figure 2. Less-protection by the protection potential result from CIPS measurement

To sum up, this study simulated the potential distribution on the pipeline by the COMSOL software and the corrected physical-electrochemical model. The protection potential result from simulation was agree with it from CIPS measurement, indicating that the method of numerical simulation by COMSOL was effective for the study about the cathode protection on the natural gas pipeline.

\section{Optimization of cathode protection system}

\subsection{Current status}

Nan 21 protection area contained Nan 3 and Nan 4 main pipelines, and other pipelines, protecting by the Nan 18 
and Nan 21 cathode protection stations (Figure 3).

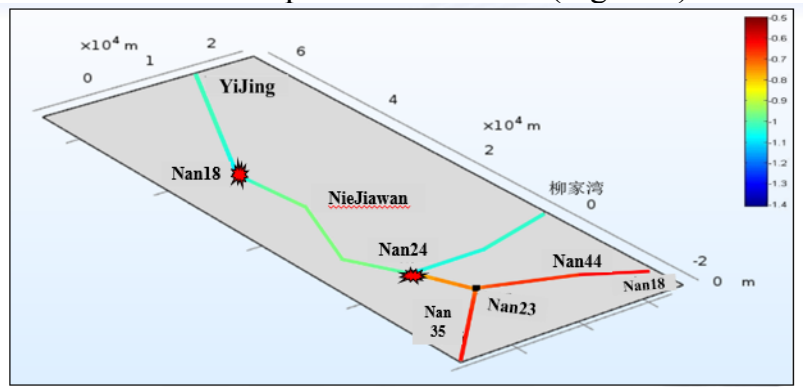

Figure 3. General view of protection area for Yijing (Nan 18 to Nan 21)
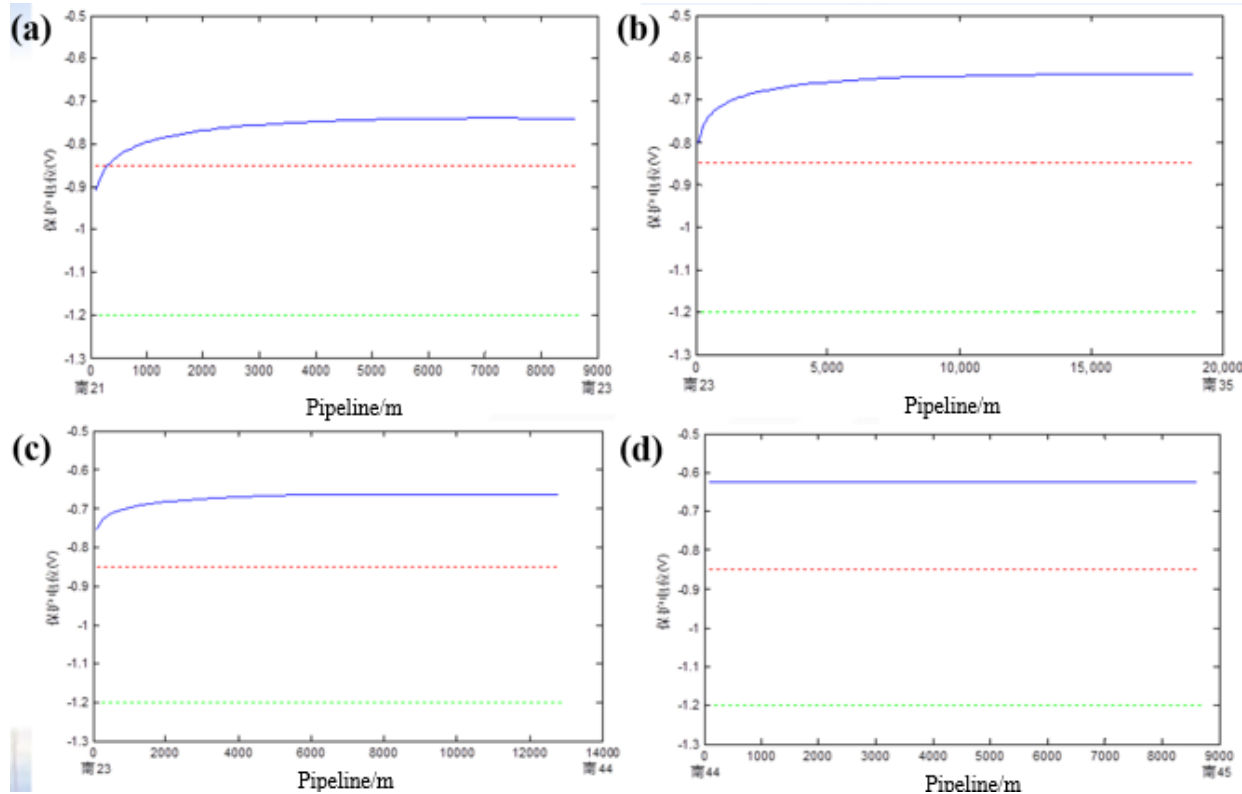

Figure 4. Potential distributions of the Nan 21 to Nan 23 branch pipeline (a), the Nan 23 to Nan 35 branch pipeline (b), the Nan 23 to Nan 44 branch pipeline (c), and the Nan 44 to Nan 45 branch pipeline (d)

\subsection{Optimizing solution}

Given to the less-protection status for the Nan 21 to Nan 23 branch pipeline and their upstream pipelines due to the massive pipelines, it was necessary to build a new cathode protection station at Nan 23 station, which could together with the Nan 21 cathode protection station to improve the protection effect for the upstream pipelines of Nan 21 branch pipeline. The optimized arrangement of cathode protection station for the area of Nan 18 to Nan 21 was shown in Figure 5.
According to the above corrected physical-electrochemical model, the protection potentials of Nan 21 to Nan 23 branch pipeline, Nan 23 to Nan 35 branch pipeline, Nan 23 to Nan 44 branch pipeline and Nan 44 to Nan 45 branch pipeline were simulated as shown in Figure 4. It could be found that all the simulated branch pipelines were in a less-protection status.

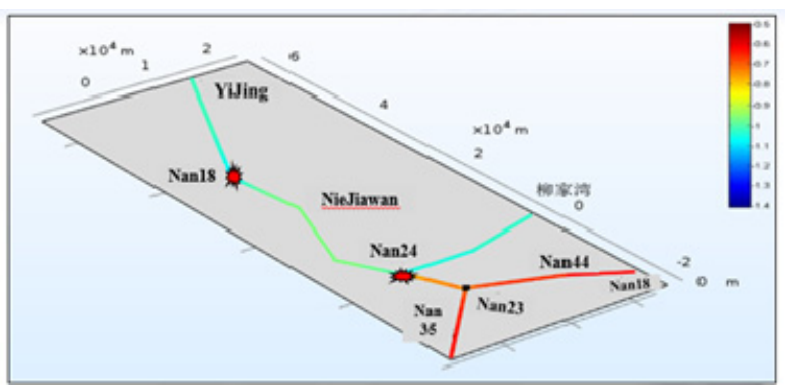

Figure 5. Optimized arrangement of cathode protection stations in the area of Nan 18 to Nan 21

After optimizing, the protection potentials of Nan 21 to Nan 23 branch pipeline, Nan 23 to Nan 35 branch pipeline, Nan 23 to Nan 44 branch pipeline and Nan 44 to Nan 45 branch pipeline were located in the acceptable zone (Figure 6), suggesting that the optimized cathode protection station could protect all the pipelines effectively around the area of Nan 18 to Nan 21 . 

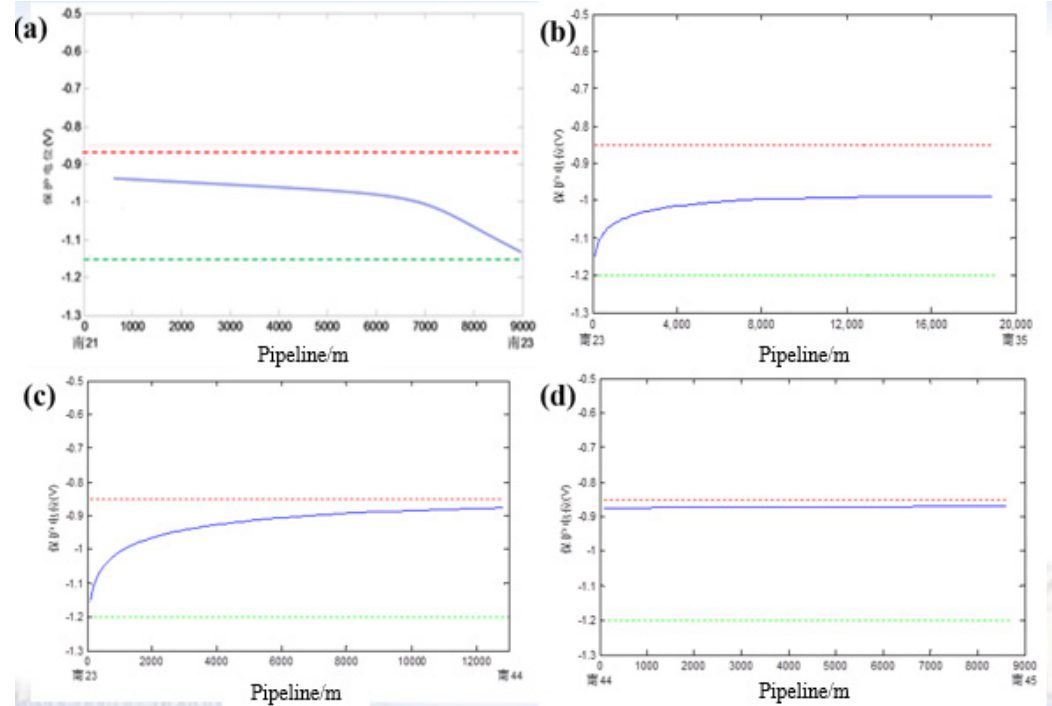

Figure 6. Potential distribution of the Nan 21 to Nan 23 branch pipeline (a), the Nan 23 to Nan 35 branch pipeline (b), the Nan 23 to Nan 44 branch pipeline (c), and the Nan 44 to Nan 45 branch pipeline (d) after optimization

\section{Conclusion}

In summary, the potential distribution of the protected pipeline was simulated based on a physical-electrochemical model, which was constructed through the irregular net-dividing for the geometric model, establishing a boundary conditions and reactions for the electrochemical model using the COMSOL software. It was found that the simulation was effective because the result from simulation was agree with that of measurement. The potential distribution from simulation for the three protected areas suggested that most pipelines were in a normal status, while there were few pipelines in a status of less-protection. However, the all pipelines were protected by the cathode protection stations after adding three cathode protection stations at the Nan 23 station through the optimization by COMSOL software. Therefore, the simulation could analyze the dynamic protection potential of the pipeline, check the running of cathode protection station, then ensuring the effectiveness of protection for the pipelines by cathode protection station. With the application of intelligence test pipe and the update of constant potential rectifier with a function of data remote transmission, the protection of pipeline could combine the dynamic simulation, live transmission and remote monitoring. It could reduce the burden on the monitoring and analysis of dynamic protection potential by integrating the software modules and data transmission into the corrosion monitoring platform-cathode protection station of the first gas plant.

\section{Acknowledgments}

The work was financially supported by the Science and Technology Plan Projects of State Administration for Market Regulation (2019MK136), CSEI Research Program (2019-Youth-03), and Research on regular inspection strategy of natural gas purification plant pipeline (K-19-D02)

\section{References}

1. Jia, C.Z., Zhang, Y.F., Zhao, X. (2014) Prospects of and challenges to natural gas industry development in China. Natural Gas Industry, 34: 8-18.

2. Zhang, Y.C., Xu, M., Liu, X., et al. (2013) Corrosion perforation failure analysis of a natural gas pipeline. Total Corrosion Control, 27: 65-67.

3. Huang, K., Wu, J.L., Quan, K., et al. (2018) Areview on evaluation technology of oil-gas pipelines with corrosion defect. Surface Technology, 47: 116-123.

4. Huang, K., Li, W., Fu, Z.H., et al. (2014) Risk assessment of $\mathrm{Ku}$-shan pipeline with the Kent-revised method. Industrial Safety and Environmental Protection, 40: 54-57.

5. Yu, S.R., Li, J.H., Li, S.X., et al. (2008) Probability model for the prediction of corrosion remaining life of underground pipelines. China Safety Science Journal, 18: 11-15.

6. Chen, D.B., Han, D.F., Ma, J.Y., et al. (2015) Prediction analysis of remaining life and plane frame structure displacement reliability of corroded pipelines based on VB/GM(1,1)/Matlab. Corrosion \& Protection, 36: 197-200.

7. Zhang, X.S., Cao, N.N., Li, Y.Y. (2016) Residual life prediction of buried oil and gas pipelines based on Gumbel extreme value type I distribution. Journal of Chinese Society for Corrosion and Protection, 36: 370-374. 